\title{
Dark matter in elliptical galaxies: prospects for WFOS/TMT
}

\author{
Patrick Côté ${ }^{1}$, Eric W. Peng ${ }^{1}$ \\ and Jarle Brinchmann ${ }^{2}$ \\ ${ }^{1}$ Herzberg Institute of Astrophysics, National Research Council of Canada, \\ 5071 W. Saanich Road, Victoria, BC V9E 2E7, Canada \\ email: Patrick.Cote@nrc-cnrc.gc.ca, Eric.Peng@nrc-cnrc.gc.ca \\ ${ }^{2}$ Centro de Astrofisica da Universidade do Porto, Rua das Estrelas, 4150-762 Porto, Portugal \\ email: jarle@astro.up.pt
}

\begin{abstract}
The amount and spatial distribution of dark matter in elliptical galaxies are poorly known, despite extensive observations with multi-object spectrographs on 4-10m-class telescopes. We examine the prospects for measuring the structure of dark matter halos in elliptical galaxies - and the orbital properties of their planetary nebula and globular cluster systems from radial velocity measurements made with WFOS, a wide-field optical spectrograph operating on the Thirty Meter Telescope (TMT).
\end{abstract}

Keywords. instrumentation: spectrographs — techniques: radial velocities — planetary nebulae - galaxies: star clusters — cosmology: dark matter

\section{Introduction: Testing cosmological models on galaxy scales}

Satellite measurements of temperature fluctuations in the cosmic microwave background have provided a spectacular confirmation of the standard cosmological model: i.e., a spatially flat, isotropic and homogeneous universe that is composed of ordinary matter, dark energy and dark matter (Spergel et al. 2003). The existence of this latter component - which may comprise as much as $90 \%$ of the mass in the universe - gained widespread acceptance by the astronomical community in the mid 1970s. But despite three decades of study, the nature of dark matter remains a mystery.

Dark matter is detectable only through its gravitational effects, and through gravity it controls the formation of virtually all structures in the universe from superclusters down to the smallest galaxies. At present, the dominant paradigm for structure formation is the family of cold dark matter (CDM) models, in which the random motions of the dark matter particles are assumed to be much slower than the speed of light. It is well known that these models provide a remarkably accurate description of the universe on scales larger than $\sim 1 \mathrm{Mpc}$. However, these same models fail by a wide margin to match observations on the much smaller scales of galaxies (see, e.g., Sellwood \& Kosowsky 2001). According to the models, the Milky Way should be enveloped in a swarm of a hundred or more low-mass galaxies, yet exhaustive searches have uncovered only a few dozen such objects. Equally perplexing, kinematic observations of galaxies suggest that the inner density profiles of their dark matter halos are significantly shallower than the model predictions.

It is unclear if this latter discrepancy represents a failure of the models or limitations in the observations. It is certainly true that attempts to measure dark matter mass profiles for galaxies have produced strongly conflicting results, with claims ranging from dark matter halos that fall steeply with distance from the galaxy center (as expected in CDM 


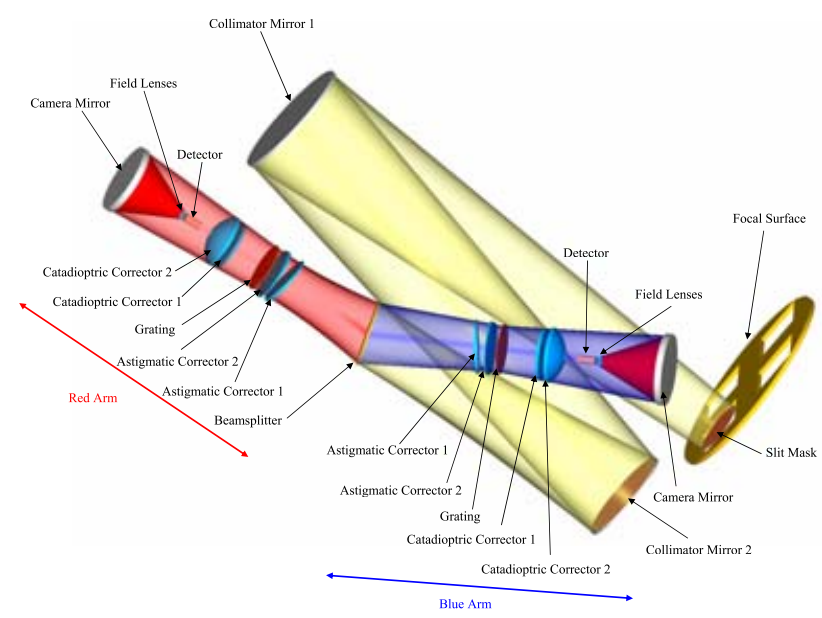

Figure 1. Schematic representation of one possible WFOS design including the separate red and blue arms. For clarity, only one of the four WFOS channels is shown. The various optical and mechanical elements have been labeled.

models) to dark matter halos with constant-density cores (at odds with the theoretical predictions). But almost all conclusions on the structure of dark matter halos rest on observations of the same kind of galaxies: flattened, gas-rich spirals. Very little is known about the dark matter distribution in elliptical galaxies, which are common in the field and which dominate rich galaxy clusters. The reason is simple: unlike spirals, ellipticals contain little or no cold gas, making the measurement of HI rotation curves impossible.

In this article, we describe an observational program for the Wide Field Optical Spectrograph (WFOS) on the Thirty Meter Telescope (TMT) which has been designed to measure the distribution of dark matter in nearby elliptical galaxies using radial velocities of dynamical "test particles" orbiting in their gravitational potential wells. The properties of the dark matter halos can then be studied through their gravitational influence on the motions of the embedded test particles.

\section{Design of the wide field optical spectrograph}

WFOS is designed to perform multi-object spectroscopy and imaging of sources over a large field $\left(\geqslant 75\right.$ arcmin $\left.^{2}\right)$ with high throughput $(\geqslant 30 \%)$ and high operational efficiency. A natural-seeing instrument, it provides complete wavelength coverage from the near-UV to the near infrared. One possible design for WFOS, currently under development at the Herzberg Institute for Astrophysics, is shown in Figure 1. The instrument will occupy a fixed location on one of the TMT Nasmyth platforms and be fed directly at the Gregorian focus by the telescope beam redirected from an articulated telescope tertiary mirror. It will be able to rotate to compensate for relative field rotation. Acquisition, guiding and calibration systems will be included in the instrument through the use of an on-instrument wavefront sensor which interfaces to the telescope control system and secondary mirror. To enhance image quality, the WFOS design will incorporate an atmospheric dispersion compensator, as well as a guiding system and active optics. For reasons of efficiency, the field of view of WFOS is broken into four separate zones, each of which is fed into a separate spectrograph. Each spectrograph is, in turn, separated into red and blue camera paths using dichroic beam splitters. A thorough exploration of the scientific capabilities of this instrument has been presented in the WFOS 
Operation Concepts Definition Document (Abraham et al. 2006). Here we focus on one specific program: dynamics of elliptical galaxies and the distribution of dark matter from radial velocity measurements of globular clusters (GCs) and planetary nebulae (PNe).

\section{Dark matter in elliptical galaxies: a WFOS/TMT program}

To map out the radial profile of the dark matter mass density, $\rho(r)$, using a system of test particles — in this case, GCs and $\mathrm{PNe}$ - one must solve simultaneously for the orbital anisotropy of the system of test particles used in the dynamical analysis: $\beta(r)=1-\sigma_{\theta}^{2} / \sigma_{r}^{2} . \dagger$ This requires a minimum sample of $\approx 1000$ radial velocities (e.g., Merritt \& Tremblay 1993). If one is willing to make some assumptions about the orbital properties of the test particles (e.g., that their orbits are isotropic, $\beta \equiv 0$ ), then this requirement can be relaxed to $\approx 250$ velocities (see Côté et al. 2001).

At present, radial velocities for $\geqslant 250$ GCs have been amassed for just three elliptical galaxies: M49, M87 and NGC1399 (Côté et al. 2001, 2003; Richtler et al. 2003). These were obvious first targets for multi-object spectrographs on $8 \mathrm{~m}$-class telescopes since they are among the nearest ellipticals with rich GC systems. But for these same reasons, they constitute a biased sample in terms of luminosity and environment: i.e., they are some of the brightest galaxies in the Local supercluster and occupy unique locations in the highest density regions of the Virgo and Fornax Clusters. The situation is comparable in the case of PNe: in only two galaxies - NGC5128 (Peng et al. 2004) and NGC4697 (Méndez et al. 2001) — does a sample of more than 250 PNe velocities exist.

The existing observations for these galaxies - along with those for a handful of other galaxies having still smaller radial velocity samples of GCs and PNe - paint a confusing picture about the structure of dark matter halos. Some galaxies appear to be dark matter dominated at their effective radius, $r_{e}$, while others seem to show no evidence at all for dark matter out to $r \lesssim 4 r_{e}$ (see the review in Napolitano et al. 2005). To elucidate the shape and extent of the dark matter halos in elliptical galaxies, and to examine the dependence of halo properties on galaxy environment, a radial velocity survey of thousands of GCs and PNe in a much expanded sample of galaxies is required.

Table 1. Globular Clusters in Elliptical Galaxies

\begin{tabular}{cccc}
\hline $\begin{array}{c}V_{\lim } \\
(\mathrm{mag})\end{array}$ & $\begin{array}{c}N_{\mathrm{GC}} \\
\geqslant 250\end{array}$ & $\begin{array}{c}\left\langle\sigma_{0}\right\rangle \\
\left(\mathrm{km} \mathrm{s}^{-1}\right)\end{array}$ \\
\hline 22.7 & 18 & 4 & $203 \pm 34$ \\
25.5 & 179 & 49 & $163 \pm 28$ \\
\hline
\end{tabular}

Table 1 compares the number of elliptical galaxies from the Catalog of Nearby Galaxies (Tully \& Fisher 1988) for which radial velocity surveys to a limiting magnitude of $V_{\lim }=$ 22.7 (e.g., Côté et al. 2001, 2003) would yield minimum radial velocity samples of 250 and 1000 GCs. Note that this is the approximate limiting magnitude for GC radial velocity surveys of M87 and M49 carried out with Keck/LRIS. Table 1 shows that, at the present time, samples of this size can be collected for 18 and 4 ellipticals, respectively. With the limiting magnitude of $V_{\lim } \approx 25.5$ expected with WFOS, these samples increase by an order of magnitude: to 179 and 49 galaxies, respectively. Similarly dramatic gains are found for PNe. In short, WFOS opens a realm of study which will never be accessible

$\dagger$ Here $\sigma_{\theta}$ and $\sigma_{r}$ are the intrinsic velocity dispersions in the tangential and radial directions relative to the galaxy center. 

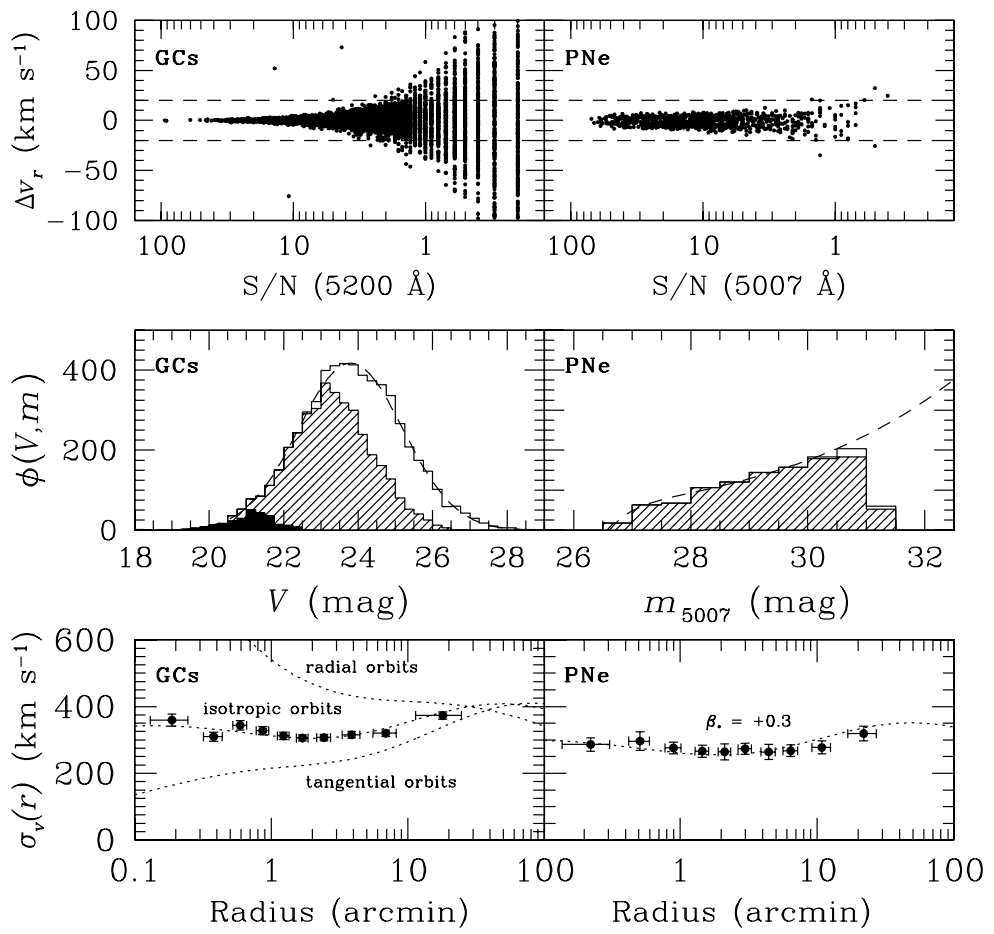

Figure 2. (Upper Panels) Difference between input and measured radial velocity for simulated GCs and PNe in M49, plotted as a function of signal-to-noise ratio. The dashed lines show the maximum acceptable velocity error. (Middle Panels) Input luminosity function of GCs (left) belonging to M49 (dashed curve and open histogram). The hatched histogram shows $\approx 4000$ GCs for which a 1 hour exposure with WFOS would yield $\mathrm{S} / \mathrm{N} \geqslant 1$. The luminosity function of the 263 GCs with existing velocity measurements from Keck is shown as the dark histogram. Input luminosity function of PNe in M49 (dashed curve and and open histogram; right). The hatched histogram shows the $\approx 1100$ PNe for which a 1 hour exposure with WFOS would yield $\mathrm{S} / \mathrm{N} \geqslant 1$. (Lower Panels) A simulated measurement of the anisotropy parameter, $\beta \equiv 1-\sigma_{\theta}^{2} / \sigma_{r}^{2}$, for the GC system of M49 (left). Different anisotropy parameters are shown by the different curves, from strongly radial $(\beta=+0.99$, upper curve), to strongly tangential $(\beta=-99)$ orbits. The measured velocity dispersion profile recovers the assumed isotropic velocity distribution $(\beta=0)$ with high confidence. For comparison, the simulated velocity dispersion profile for $\mathrm{PNe}$ is shown in the right panel. In this case, the dashed curve shows the velocity dispersion profile for the assumed (input) case of mildly radial stellar orbits, $\beta_{*}=+0.3$.

with multi-object spectrographs on $8-10 \mathrm{~m}$ class telescopes. Note that the final column of Table 1 gives an estimate of the mean projected central velocity dispersion, $\left\langle\sigma_{0}\right\rangle$. This sets the required velocity precision: $\epsilon_{v}$ must be less than $20 \mathrm{~km} \mathrm{~s}^{-1}$ for the uncertainties on individual velocity measurements to be no more than $\approx 0.1$ times the system's intrinsic velocity dispersion.

\subsection{A Case Study: Observations of GCs and PNe in M49 (NGC4472)}

GC systems have a near-Gaussian magnitude distribution, with $\mathrm{M}_{V} \approx-7.4$ and dispersion $\approx 1.4$ mag. At the distance of M49, the brightest member of the Virgo Cluster, the GC luminosity function "turns over" at $\mathrm{V} \approx 23.8$. With limiting magnitudes of $\mathrm{V}_{\lim } \approx 22.7$, existing radial velocity surveys with $8 \mathrm{~m}$-class telescopes have sampled only a tiny fraction of the GC luminosity function (see Figure 2). The surface density of GCs varies sharply 
over the face of the galaxy: from $\Sigma_{\mathrm{GC}} \geqslant 100 \mathrm{arcmin}^{-2}$ in the core, to $\Sigma_{\mathrm{GC}} \sim 1 \mathrm{arcmin}^{-2}$ beyond $10^{\prime}$.

By contrast, the PN luminosity function in M49 is expected to show an abrupt onset at $\mathrm{m}_{5007} \approx 26.6$ and a sharp rise to fainter magnitudes. Since the distribution of PNe follows the brightness profile of the underlying galaxy, the surface density of sources also varies significantly with radius. For sources brighter than $\mathrm{m}_{5007} \approx 31$ - the approximate limiting magnitude for WFOS in a single 1 hour exposure - the mean surface density is $\Sigma_{\mathrm{PN}} \sim 16 \operatorname{arcmin}^{-2}$ within the galaxy's effective radius $\left(\mathrm{r}_{e}=3.5\right)$.

For both the GCs and PNe, a "nod and shuffle" technique is recommended to maximize slit density and boost observing efficiency. In both cases, program objects would be observed simultaneously in the blue and red arms with resolutions of $\mathcal{R} \approx 2000$ or 5000 . Monte Carlo simulations of GC/PN spectra based on 1-hour integrations with WFOS, in $0 . \prime 8$ seeing and dark sky conditions, show that this observational strategy is adequate to ensure a velocity precision $\lesssim 20 \mathrm{~km} \mathrm{~s}^{-1}$ for most GCs and PNe.

\subsection{Measuring the $\beta(r)$ for the $M 49 G C$ and $P N$ systems}

Figure 2 summarizes our findings from Monte Carlo simulations of WFOS spectra for GCs and PNe in M49. These simulations suggest that, in $\approx 20$ hours of "open shutter" observing time, it would be possible to measure radial velocities for $\approx 4000$ GCs and $\approx 1100$ PNe. The upper panels of Figure 2 show the difference between input and recovered radial velocity for the simulated GCs/PNe; the middle panels of this figure show their respective luminosity functions. In the lower panels, we plot velocity dispersion profiles of the GCs and PNe, $\sigma_{v}(r)$, along with model predictions for different orbital anisotropies. The input anisotropies - $\beta(r)=0$ and +0.3 for the GCs and PNe, respectively - are recovered with high confidence. Because the measurement of $\rho(r)$ requires a simultaneous measurement of $\beta(r)$, it is clear that WFOS has the potential to open a new realm in the study of dark matter and elliptical galaxy dynamics.

\section{Acknowledgements}

The authors thank the members of the WFOS science and engineering teams for helpful discussions and suggestions.

\section{References}

Abraham, B., et al. 2006, TMT WFOS Operational Concepts Definition Document

Côté, P., McLaughlin, D.E., Hanes, D.A., Bridges, T.J., Geisler, D., Merritt, D., Hesser, J.E., Harris, G.L.H. \& Lee, M.G. 2001, ApJ 559, 828

Côté, P., McLaughlin, D.E., Cohen, J.G. \& Blakeslee, J.P. 2003, ApJ 591, 850

Méndez, R.H., Riffeser, A., Kudritzki, R.-P., Matthias, M., Freeman, K.C., Arnaboldi, M., Capaccioli, M. \& Gerhard, O.E.. 2001, ApJ 563, 135

Merritt, D. \& Tremblay, B. 1993, AJ 106, 229

Napolitano, N.R., Capaccioli, M., Romanowsky, A.J., Douglas, N.G., Merrifield, M.R., Kuijken, K., Arnaboldi, M., Gerhard, O. \& Freeman, K.C 2005, MNRAS 357, 691

Peng, E.W., Ford, H.C. \& Freeman, K.C. 2004, ApJ 602, 705

Richtler, T., Dirsch, B., Gebhardt, K., Geisler, D., Hilker, M., Alonso, M.V., Forte, J.C., Grebel, E. K., Infante, L., Larsen, S., Minniti, D. \& Rejkuba, M. 2004, AJ 127, 2094

Sellwood, J.A., \& Kosowsky, A. 2001, in: J. Hibbard, M. Rupen \& J. van Gorkom (eds.), ASP Conf. Ser. 240, Gas and Galaxy Evolution, (San Francisco: ASP), 311

Spergel, D.N., et al. 2003, ApJS 148, 175

Tully, R.B. \& Fisher, J.R. 1988, Nearby Galaxies Catalog (Cambridge: Cambridge Univ. Press) 